\title{
Recomendação e Agregação de Conteúdos Relacionados em conformidade com o padrão SCORM
}

\author{
Daniel E. Neves, Wladmir C. Brandão, Lucila Ishitani \\ Programa de Pós-Graduação em Informática - Pontifícia Universidade Católica de \\ Minas Gerais (PUC Minas) CEP 31980110 - Belo Horizonte - MG- Brasil \\ den.net@hotmail.com, \{ wladmir, lucila\}@pucminas.br
}

\begin{abstract}
In this work we proposed a methodology for automatic recommendation and aggregation of related Learning Objects in SCORM standard. Differently from other approaches in the literature, our proposal uses the metadata of the category "relation" to aggregate content without extending these metadata, or changing SCORM, or even developing specific implementations on the Learning Management System (LMS), keeping compatibility of the content package with any LMS that offers support to the standard.
\end{abstract}

Resumo. O presente trabalho apresenta uma metodologia para recomendação automática e agregação de Objetos de Aprendizagem (OAs) relacionados, conforme o padrão SCORM. Diferentemente de outras abordagens na literatura, são utilizados metadados da categoria "relation" para agregação de conteúdos, sem a necessidade de extensão desses metadados, de realizar alterações no padrão, ou mesmo de implementações especificas nos Sistemas de Gerenciamento de Aprendizagem (SGA), mantendo a compatibilidade do conteúdo com qualquer SGA que ofereça suporte ao padrão.

\section{Introdução}

O presente trabalho apresentada uma metodologia que emprega técnicas de recuperação da informação e mineração de textos para recomendação automática e agregação de Objetos de Aprendizagem (OAs) relacionados, conforme o padrão denominado Sharable Content Object Reference Model (SCORM). Foi utilizado o vocabulário da categoria de metadados relation, definida pelo padrão, para identificar relações dos tipos requires e isrequiredby, ispartof e haspart, references e isreferencedby, isbasedon e isbasisfor, sem a necessidade de extensão de seus metadados, alterações no SCORM ou implementações específicas no Sistema de Gerenciamento de Aprendizagem (SGA), diferentemente de outros trabalhos referenciados na literatura. Foi implementado o protótipo de um sistema computacional, que aplica a metodologia proposta sobre uma amostragem de OAs e gera os resultados necessários à avaliação de sua eficácia. Os resultados obtidos são positivos e foram avaliados com o apoio de profissionais da pedagogia que atuam no desenvolvimento de conteúdos para e-Learning.

Este trabalho está organizado da seguinte forma: a Seção 2 apresenta os trabalhos relacionados; a Seção 3, a metodologia proposta; a Seção 4, a avaliação e os resultados e a Seção 5, as conclusões e indicação de trabalhos futuros. 


\section{Trabalhos Relacionados}

Roy, Sudeshna e Sujoy (2008) e Engelhardt e outros (2006) apresentam abordagens bastante consistentes e viáveis para anotação automática de OAs. Todavia, ambos utilizam extensões ao modelo de metadados do SCORM e SGAs específicos, o que reduz a compatibilidade do pacote de conteúdos frente a outros sistemas. No caso de Engelhardt e outros (2006), a solução proposta pode não ser adequada, pois leva a um acesso disperso a um conjunto de variados OAs sobre um extensa rede semântica, haja vista que estes não são recomendados, mas sim pesquisados. Em Lu e Hisieh (2009) foi desenvolvido um modelo próprio de extensão aos metadados da categoria relation, que foi implementado em Lu e outros (2010), no protótipo de um SGA, onde as relações são atribuídas manualmente. Os autores obtiveram um modelo distante do SCORM e que é tratado por um sistema que não suporta o padrão em si, mas sim um conjunto específico de definições, que não encontrarão suporte em outros SGAs. Hernández e outros (2009) desenvolveram um sistema em que a saída consiste de OAs extraídos de uma fonte de documentos. A variedade de OAs extraídos pode ser grande ou pequena, dependendo dos documentos utilizados, podendo resultar em redundância destes OAs e ocasionar um baixo aproveitamento de seu conjunto. $\mathrm{O}$ sistema não oferece recursos que auxiliem na composição de conteúdos mais extensos e complexos, que demandem a identificação e seleção de OAs relacionados entre si, capazes de oferecer conteúdos complementares e por vezes sequenciáveis, compondo, em seu conjunto, o conteúdo final.

\section{Metodologia para Recomendação e Agregação de OAs}

A metodologia proposta e avaliada neste trabalho consiste nos seguintes processos principais, que serão detalhados ao longo desta seção: a partir de uma base de conhecimento de domínio, um conjunto de OAs é submetido a um processo de recuperação de informações relevantes, identificadas e automaticamente anotadas em cada documento. A partir destas informações, são geradas e também anotadas diversas associações ontológicas para cada $\mathrm{OA}$, que indicam as possíveis relações que possam estabelecer com diferentes OAs, cujos conteúdos pertençam à mesma área de domínio. Em seguida, os OAs são armazenados em um repositório de conteúdos, a partir do qual podem ser selecionados e utilizados para composição de um dado conteúdo. Este conteúdo é submetido a um processo de recomendação de conteúdos relacionados, a partir dos OAs presentes no repositório, com base em suas associações ontológicas. Ao final deste processo, os OAs recomendados podem ser mantidos ou excluídos manualmente. Unindo-se OAs pré-selecionados e OAs recomendados, um pacote de conteúdos no formato SCORM é gerado de acordo com as especificações do SCORM.

Para recuperar informações relevantes aos conteúdos dos OAs, o sistema implementado utiliza um plugin do framewok GATE denominado ANNIE, discutido em Cunningham e outros (2012), e o módulo AssignerRelevance, implementado como parte deste trabalho. Dados como entrada um corpus, com novos OAs, e a base de conhecimento de domínio, a saída do ANNIE consiste nos OAs contendo termos e conceitos anotados quanto à sua classe gramatical e sua classificação ontológica. Estes OAs são processados pelo AssignerRelevance, que utiliza os metadados gerados pelo ANNIE e implementa os algoritmos para geração dos demais metadados, aos quais são associadas métricas necessárias ao cálculo de relevância para cada termo ou conceito anteriormente anotado. O AssignerRelevance também realiza a classificação hierárquica destes elementos, com base em sua relevância em relação ao texto como um todo, 
gerando um vetor de termos mais relevantes (VT) para cada documento. Em seguida, a próxima etapa consiste em se identificar as possíveis relações para cada OA. Para isso, foi implementado um segundo módulo do sistema, denominado AssociationsBuilder, que recebe como entrada a lista dos novos OAs presentes no corpus e a base de conhecimento de domínio, fornecendo como saída as diversar associações possíveis para cada um deles, a partir de cada elemento de VT e com base nas relações descritas pela ontologia de domínio, que obedecem ao vocabulário da categoria relation do SCORM. Cada associação gerada contém o termo relevante, sua classe, superclasse, subclasses e as relações, que têm sua classe como domínio e a classe associada como alcance, formando um grafo sobre a ontologia. As associações geradas para cada OA são neles anotadas por meio de novos metadados.

Para a recomendação e agregação de OAs relacionados, foi implementado um módulo do sistema denominado RecommendationsBuilder, que recebe como entrada uma lista de OAs, que são selecionados pelo usuário a partir do repositório. Dentre os metadados anotados em cada OA, nas etapas anteriores, se encontram as diversas associações geradas. Para cada associação encontrada, as relações que a compõem são analisadas e as classes de termos para as quais apontam como seu alcance são identificadas. Desse modo, para cada relação, presente em cada uma das associações geradas, para cada um dos OAs da lista de entrada, é realizada uma busca no repositório de conteúdos por outros OAs cujos termos mais relevantes pertençam à classe de alcance da relação em análise. Em cada OA encontrado seu VT é analisado. Caso contenha o termo fonte da associação, este OA é então recomendado como conteúdo relacionado ao OA principal e o tipo de relação é qualificada como sendo do tipo descrito na associação do termo fonte. Dentre os documentos recomendados, o usuário seleciona os que deseja manter, fazendo com que sejam agregados ao conteúdo principal através de sua inserção no pacote de conteúdo SCORM.

\section{Avaliação e Resultados}

Os conteúdos dos OAs utilizados neste trabalho pertencem ao domínio da área de conhecimento da música erudita. A base de conhecimento de domínio utilizada é composta de um dicionário de termos e de uma ontologia de domínio. As classes da ontologia e respectivas relações foram modeladas em UML. Assim, foi possível mapear as relações definidas na categoria relation em relações estabelecidas na UML, por meio de associações, agregações, heranças e especializações.

Para avaliação de nossa metodologia foi organizado um repositório de OAs, composto de 8967 documentos. A partir dele foi gerada uma amostragem, mantendo-se a mesma proporção no percentual de documentos, para cada classe, totalizando 111 OAs: 37 sobre biografias de compositores, 2 sobre períodos históricos, 14 sobre termos de glossário e 58 sobre obras musicais. Foi solicitado a três profissionais da Educação que realizassem, individualmente, a anotação manual destes OAs, indicando ao final os termos que julgassem mais relevantes a cada um deles, a partir dos quais deveriam ser recomendados outros documentos a eles relacionados, no intuito de complementar seu conteúdo. Foram utilizados para análise os dois resultados que apresentaram maior número de anotações, apresentados e comparados na Tabela 1, cujos colaboradores serão identificados como A e B. Também foi computado o número de resultados coincidentes, que consiste no conjunto interseção dos termos anotados por ambas os 
colaboradores, para cada classe de documentos. O mesmo foi feito para os termos indicados como mais relevantes.

Tabela 1 - Resultados da anotação manual

\begin{tabular}{|c|c|c|c|c|c|c|c|c|}
\hline \multicolumn{3}{|c|}{ Anotações Sobre a Amostragem } & \multicolumn{2}{|c|}{ Col. A } & \multicolumn{2}{|c|}{ Col. B } & \multicolumn{2}{|c|}{ Acurácia } \\
\hline Total & Relevantes & Acurácia & Total & Rel. & Total & Rel. & Col. A & Col. B \\
\hline 1892 & 1231 & 65,06 & 749 & 492 & 1143 & 739 & $65,69 \%$ & $64,65 \%$ \\
\hline \multirow{4}{*}{$\begin{array}{l}\text { Média de } \\
\text { Anot. / } \\
\text { documento } \\
\text { / classe }\end{array}$} & \multicolumn{2}{|c|}{ Biog. de Compositores } & 8,43 & 4,50 & 20,07 & 15,50 & $53,39 \%$ & $77,22 \%$ \\
\hline & \multicolumn{2}{|c|}{ Períodos Históricos } & 156,0 & 117,0 & 123,0 & 117,0 & $75,00 \%$ & $95,12 \%$ \\
\hline & \multicolumn{2}{|l|}{ Glossário } & 7,14 & 5,93 & 5,50 & 5,43 & $83,00 \%$ & $98,70 \%$ \\
\hline & \multicolumn{2}{|c|}{ Obras Musicais } & 4,36 & 2,81 & 6,46 & 1,90 & $64,59 \%$ & $29,40 \%$ \\
\hline \multicolumn{3}{|c|}{ Indicação de termos mais relevantes } & \multicolumn{2}{|c|}{287 termos } & \multicolumn{2}{|c|}{191 termos } & \multicolumn{2}{|c|}{478 total } \\
\hline
\end{tabular}

Foram considerados não relevantes os termos e conceitos manualmente anotados que não se referiam ao domínio da música erudita, tendo como referência principal a ontologia de domínio. Estes termos também não foram computados na contagem dos termos indicados como mais relevantes para cada documento. Percebeu-se a dificuldade em se manter a coerência no processo de anotação manual. Ao anotar documentos pertencentes à mesma classe, com o mesmo formato e padrão para disponibilização das informações, ora um determinado conjunto de termos foi marcado como relevante, ora não, pelo mesmo colaborador. Nos textos relativos às obras musicais, o colaborador $\mathrm{A}$ manteve o padrão de anotação, enquanto o colaborador B ora inseria anotações na sinopse da obra, ora na ficha técnica, ora nos nomes dos personagens. Além disso, a quantidade de anotações do colaborador B diminuiu consideravelmente entre os primeiros e últimos documentos anotados, sendo que nos documentos maiores foram indicados como termos mais relevantes apenas aqueles presentes na primeira página.

Concluído o trabalho de anotação manual, assim como a análise do material produzido, a mesma amostragem foi submetida ao protótipo implementado neste trabalho, executando-se todos os processos, da anotação à recomendação. Os resultados da anotação automática são apresentados na Tabela 2.

Tabela 2 - Resultados da anotação automática

\begin{tabular}{|c|l|c|c|c|}
\hline \multicolumn{2}{|c|}{ Anotações Sobre a Amostragem } & Total & Verdadeiros Positivos & Acurácia \\
\hline \multicolumn{2}{|c|}{ Termos Anotados: } & $\mathbf{6 2 2 8}$ & $\mathbf{4 9 8 8}$ & $\mathbf{8 0 , 0 9 \%}$ \\
\hline \multirow{2}{*}{$\begin{array}{c}\text { Média de } \\
\text { Anotações } \\
\text { /documento/ }\end{array} \begin{array}{c}\text { classe } \\
\text { classe }\end{array}$} & Biografia de Compositores & 84,93 & 71,14 & $83,77 \%$ \\
\cline { 2 - 5 } & Períodos Históricos & 1213,00 & 1083,00 & $89,28 \%$ \\
\cline { 2 - 5 } & Glossário & 23,71 & 23,07 & $97,29 \%$ \\
\hline
\end{tabular}

Percebe-se uma diferença entre os resultados automático e manual, onde no segundo não há ocorrência de termos não relevantes, devido à consistência da base de conhecimento, pois apenas termos nela presentes são anotados. Todavia, a atenção se volta para a geração de falsos positivos, devido a problemas difíceis da recuperação da informação e que fogem ao escopo deste trabalho, tais como o tratamento de homônimos e de duplicatas. É interessante atentar para o fato de que estes falsos positivos, em sua maioria, foram eliminados ou receberam ponderação muito baixa no processo de classificação hierárquica, conforme os parâmetros descritos na Seção 3.

A fase de associação processa apenas os termos anotados de maior relevância em cada documento. Sobre a amostragem utilizada foram geradas 3508 associações ontológicas sobre os 111 OAs. Para testar especificamente o processo de recomendação 
de conteúdos relacionados, foi dado como entrada o OA que contém o maior conteúdo, pertence à classe Períodos Históricos e totaliza 363 associações. As relações estabelecidas por estas associações foram analisadas pelo sistema e geraram um conjunto de 12 recomendações para este documento sobre os 111 OAs contidos na amostragem. Estas recomendações estão divididas da seguinte forma: um OA como isrequiredby; sete como isreferencedby e quatro como haspart. As 12 recomendações geradas automaticamente estavam corretas.

\section{Conclusões e Trabalhos Futuros}

Com base na avaliação realizada, observa-se que a metodologia proposta nesta pesquisa é viável e produz resultados com precisão superior aos alcançados por seres humanos. Dessa forma, a recomendação automática de OAs relacionados pode auxiliar os desenvolvedores de conteúdo para e-Learning na composição de OAs em conformidade com o SCORM, reduzindo o tempo e o esforço necessários ao desenvolvimento e agregação facilitando assim o seu reaproveitamento.

Devido ao fato de a metodologia proposta ser fortemente dependente da base de conhecimento, falsos negativos podem ocorrer para os termos e conceitos que não estão nela presentes. A identificação de novas classes na ontologia pode ampliar a cobertura para a recomendação de conteúdos relacionados sobre a área de domínio. A redução do número de falsos positivos no processo de geração e anotação de metadados é de extrema importância, pois estes termos podem impactar no final do processo, gerando falsos positivos também nos documentos recomendados.

\section{Agradecimento}

A todos que colaboraram com este trabalho. À FAPEMIG pelo apoio financeiro.

\section{Referências}

Advanced Distributed Learning. (2013) "SCORM 2004 4th Edition - Content Aggregation Model [CAM]", http://www.adlnet.gov. Acesso em: 21 abr. 2013.

Engelhardt, Michael et al. (2006) "Reasoning about eLearning Multimedia Objects". In Proceedings of the International Workshop on Semantic WEB Annotations for Multimedia. http://citeseerx.ist.psu.edu/viewdoc/summary?doi=10.1.1.120.8484. Acesso em: 9 nov. 2013.

Cunningham, Hamish et al. (2012) "Developing Language Processing Components with GATE Version 7 (a User Guide)". University of Sheffield Department of Computer Science, Disponível em: <https://gate.ac.uk/sale/tao/tao.pdf>. Acesso em: 11 jul. 2013.

Hernández, Alvaro et al. (2009) "Convirtiendo el contenido de archivos en objetos de aprendizaje”. XX Simpósio Brasileiro de Informática na Educação. Disponível em: $<$ http://www.niee.ufrgs.br/eventos/SBIE/2009/conteudo/artigos/completos/62173_1.pdfs. Acesso em: 8 set. 2014.

$\mathrm{Lu}$, Eric Jui-Lin and Hsieh, Chin-Ju. (2009) "A relation metadata extension for SCORM Content Aggregation Model”. Computer Standards \& Interfaces, v. 31, p. 1028-1035, set.

$\mathrm{Lu}$, Eric Jui-Lin et al. (2010) "Extended relation metadata for SCORM-based learning content management systems". Educational Technology \& Society, v. 13, p. 220-235, jan. Disponível em: <http://www.ifets.info/journals/13_1/21.pdf >. Acesso em: 21 abr. 2013.

Roy, Devshri, Sudeshna, Sarkar and Sujoy Ghose. (2008) "Automatic extraction of pedagogic metadata from learning content". International Journal of Artificial Intelligence in Education, v. 18 , p. $97-118$. abr. 